\title{
Atherosclerotic metabolites: basic science is progressing, so need to think about clinical implications.
}

Taavi Tillmann, Centre for Non-Communicable Disease, Institute for Global Health, University

College London t.tillmann@ucl.ac.uk

This editorial refers to 'Serum metabolic signatures of coronary and carotid atherosclerosis and subsequent cardiovascular disease', by I Tzoulaki et al.

Metabolites are small molecules with a molecular weight of less than 1500 kilo daltons. This includes over 40000 different kinds of amino acids, fatty acids, sugars and antibiotics, but excludes larger proteins such as enzymes, cytokines, interleukins and C-Reactive Protein. Metabolomics is the study of a wide set of metabolites with a single test. Various laboratory techniques have been developed to generate metabolomic data. Associating these to disease outcomes in humans is a new and rapidly developing area of research. The first seminal studies that did this employed Mass Spectrometry equipment, ${ }^{23}{ }^{4}$ while more recently the less expensive proton nuclear magnetic resonance (NMR) equipment has also been used successfully. ${ }^{56}$ Usually, such analyses of metabolomic data generated from NMR equipment has relied heavily on the manufacturer's default processes, which convert data from thousands of spectra into a few hundred identified metabolites. Few studies have gone back to the raw spectral data, and tried to associate these spectral features to disease outcomes in a fully untargeted manner.

In this context, the study by Tzoulaki et al. in the current issue of the European Heart Journal is remarkably novel. In their discovery analyses, the authors regressed 30'590 metabolomic spectral features against two cardiovascular phenotypes. Those spectra with the smallest P-values were tagged. Half of the tagged metabolites could be identified, as belonging to a named metabolite. Those metabolites that failed to replicate nominally in the validation cohort $(\mathrm{P}<0.05)$ were removed. Following this, the authors find 17 metabolites associated with coronary artery calcium (CAC) or carotid intima-media thickness (IMT), plus 12 further metabolites associated with CAC and IMT. This total number of discovered and validated metabolites is far in exceedance of that reported by previous studies, ${ }^{567}$ perhaps on account of three reasons. First, the outcome phenotypes were continuous and cross-sectional (as opposed to CVD incidence, which is less frequent). Second, there were at least 3000 people in the both the discovery and validation cohorts. Third, the authors performed significance testing prior to metabolite identification, which may have led to statistical advantages. Altogether, this serves as an exemplar of how to conduct good discovery work.

The next big question will be to what degree these results can be replicated by others. After scanning similar papers produced by others, there are grounds for remaining pessimistic about whether rigorous replication will ever be performed, as it is always more exciting to discover than to replicate.

Nonetheless, in order for the field to progress to its next natural stage, it is important for researchers to come together and seek to integrate the various findings that have been created by a diversity of methods (including differences in equipment, statistical approaches, and phenotype selection). This will help pinpoint the most useful signals from a small but nonetheless noisy literature that has evolved so far.

It is important to keep in mind how this research agenda might hope to improve clinical or preventative care in the future. A common hope is to discover new metabolic risk factors that are both causal and fully independent of the established cardiovascular (CVD) risk factors (green arrow in summary Figure). In this regard, the study by Tzoulaki et al. offers grounds for scepticism. In this 
study, most of the discovered associations between metabolites and atherosclerotic phenotypes had attenuated by more than half in magnitude, after statistically adjusting for the role of conventional cardiovascular risk factors. While such observational comparisons are by no means definitive, this nonetheless raises the possibility that many of the discovered metabolites are actually mediators, that lie further downstream of established cardiovascular risk factors (blue arrow in summary Figure). If this turns out to be true, then it might focus the translational attention towards a second hope: as we wait for CVD risk factors to decline in the general population, harm could be mitigated in the interim by novel pharmaceuticals that disrupt metabolic mediators that lie in between established cardiovascular risk factors and cardiovascular disease. Mendelian randomization studies might be well placed in the future, to best explore the causality from risk factor, to metabolite, to CVD. A third translational hope is also worth considering. Even if all these metabolites were found to be non-causal markers, they may still enhance risk prediction. It is perfectly valid for risk prediction algorithms to include noncausal risk factors, particularly when their measurement error is smaller than the causal risk factors that they mark. For example, we know that some factors like alcohol are difficult to measure accurately by self-report. Metabolomic proxies of alcohol consumption may prove to be quick, cheap and acceptable way of enhancing existing CVD risk prediction models. ${ }^{89}$

In summary, this is an exemplary discovery paper. This body of literature would now benefit from the synthesis of its various findings, and how it could improve the prevention or treatment of atherosclerotic disease.

Conflict of interest: none declared.

Take home figure: Possible pathways of association between conventional cardiovascular risk factors, metabolites, and atherosclerosis.

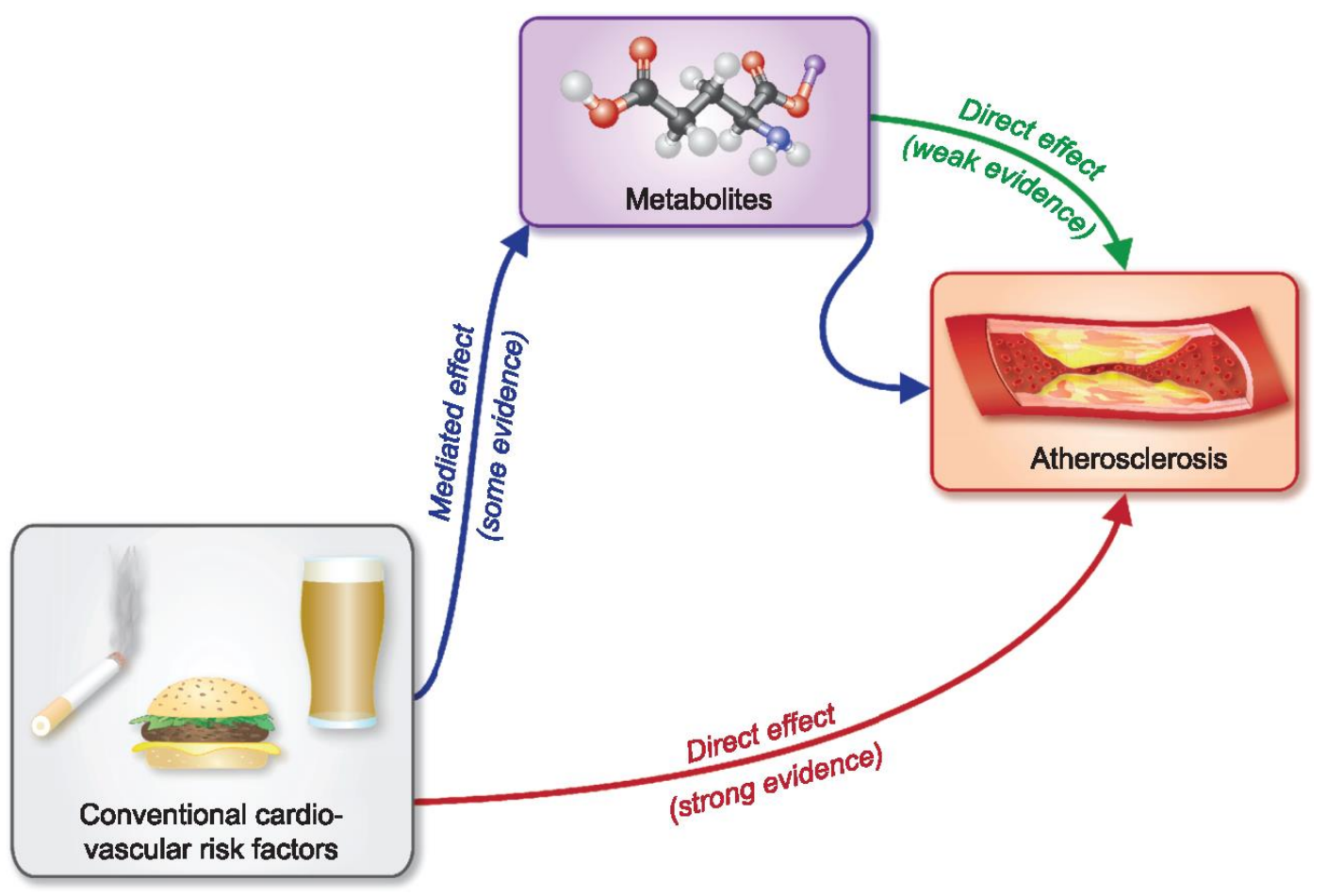




\footnotetext{
${ }^{1}$ Tzoulaki I et al. Serum metabolic signatures of coronary and carotid atherosclerosis and subsequent cardiovascular disease. Eur H J. 2019.
}

${ }^{2}$ Wang Z, Klipfell E, Bennett BJ, Koeth R, Levison BS, DuGar B, Feldstein AE, Britt EB, Fu X, Chung YM, $\mathrm{Wu}$ Y. Gut flora metabolism of phosphatidylcholine promotes cardiovascular disease. Nature. 2011 Apr;472(7341):57.

${ }^{3}$ Wang TJ, Larson MG, Vasan RS, Cheng S, Rhee EP, McCabe E, Lewis GD, Fox CS, Jacques PF, Fernandez C, O'donnell CJ. Metabolite profiles and the risk of developing diabetes. Nature medicine. 2011 Apr;17(4):448.

${ }^{4}$ Shah SH, Sun JL, Stevens RD, Bain JR, Muehlbauer MJ, Pieper KS, Haynes C, Hauser ER, Kraus WE, Granger CB, Newgard CB. Baseline metabolomic profiles predict cardiovascular events in patients at risk for coronary artery disease. American heart journal. 2012 May 1;163(5):844-50.

${ }^{5}$ Fischer K, Kettunen J, Würtz P, Haller T, Havulinna AS, Kangas AJ, Soininen P, Esko T, Tammesoo ML, Mägi R, Smit S. Biomarker profiling by nuclear magnetic resonance spectroscopy for the prediction of all-cause mortality: an observational study of 17,345 persons. PLoS medicine. 2014 Feb 25;11(2):e1001606.

${ }^{6}$ Würtz P, Havulinna AS, Soininen P, Tynkkynen T, Prieto-Merino D, Tillin T, Ghorbani A, Artati A, Wang Q, Tiainen M, Kangas AJ. Metabolite Profiling and Cardiovascular Event Risk: A Prospective Study of 3 Population-Based Cohorts. Circulation. 2015 Mar 3;131(9):774-85.

${ }^{7}$ Würtz P, Raiko JR, Magnussen CG, Soininen P, Kangas AJ, Tynkkynen T, Thomson R, Laatikainen R, Savolainen MJ, Laurikka J, Kuukasjärvi P. High-throughput quantification of circulating metabolites improves prediction of subclinical atherosclerosis. European heart journal. 2012 Mar 26;33(18):2307-16.

${ }^{8}$ Harada S, Takebayashi T, Kurihara A, Akiyama M, Suzuki A, Hatakeyama Y, Sugiyama D, Kuwabara K, Takeuchi A, Okamura T, Nishiwaki Y. Metabolomic profiling reveals novel biomarkers of alcohol intake and alcohol-induced liver injury in community-dwelling men. Environmental health and preventive medicine. 2016 Jan;21(1):18.

${ }^{9}$ Würtz P, Cook S, Wang Q, Tiainen M, Tynkkynen T, Kangas AJ, Soininen P, Laitinen J, Viikari J, Kähönen M, Lehtimäki T. Metabolic profiling of alcohol consumption in 9778 young adults. International journal of epidemiology. 2016 Aug 5;45(5):1493-506. 